\title{
Power Consumption in Telecommunication Networks: Overview and Reduction Strategies
}

\author{
Willem Vereecken, Ward Van Heddeghem, Margot Deruyck, Bart Puype, Bart Lannoo, \\ Wout Joseph, Member, IEEE, Didier Colle, Member, IEEE, Luc Martens, Member, IEEE and \\ Mario Pickavet, Member, IEEE \\ Ghent University - IBBT, Department of Information Technology (INTEC) \\ Gaston Crommenlaan 8, Bus 201, 9050 Ghent, Belgium \\ email: Willem.Vereecken@intec.ugent.be \\ web: www.intec.ugent.be
}

\begin{abstract}
One of the main challenges for the future of information and communication technologies is the reduction of the power consumption in telecommunication networks. The key consumers are the home gateways at the customer premises for fixed line access technologies and the base stations for wireless access technologies. However, with increasing bit rates, the share of the core networks could become significant as well. In this paper we characterize the power consumption in the different types of networks and discuss strategies to reduce the power consumption.
\end{abstract}

\section{INTRODUCTION}

In the last decade the attention for environment-friendly solutions has drastically increased. Especially due to the debate concerning climate change every emerging technology is scrutinously evaluated on its carbon footprint. This is also the case for information and communication technologies (ICT). It is estimated that ICT is accountable for $2-4 \%$ of the worldwide carbon emissions. The power consumption during the use phase of the equipment accounts for roughly $40-$ $60 \%$ of the carbon emissions. By 2020 these emissions are expected to double if no initiatives are taken to reduce this footprint. A significant part of these emissions, about one sixth, is attributed to telecommunication networks [1].

Worldwide, the growth rate of internet users is about $20 \%$ per year. In developing countries this growth rate is closer to $40-50 \%$. Thus, the share of greenfield deployments in telecommunication networks will be significant. Therefore emerging technologies need to be evaluated on their environmental impact. Also, ICT is being regarded as a solution with the potential to eliminate about $15 \%$ of the global carbon footprint [2]. If the sector wishes to realize its ambitions, it will also need to demonstrate it is capable of reducing its own footprint.

The work described in this paper was carried out with the support of the BONE project ("Building the Future Optical Network in Europe"), a Network of Excellence funded by the European Community's Seventh Framework; the IBBT-project GreenICT and the STRONGEST project funded by the European Community's Seventh Framework Programme FP7/2007-2013 under grant agreement $\mathrm{n}^{\circ} 247674$

W. Joseph is a Post-Doctoral Fellow of the FWO-V (Research FoundationFlanders).

Manuscript received April, 2010

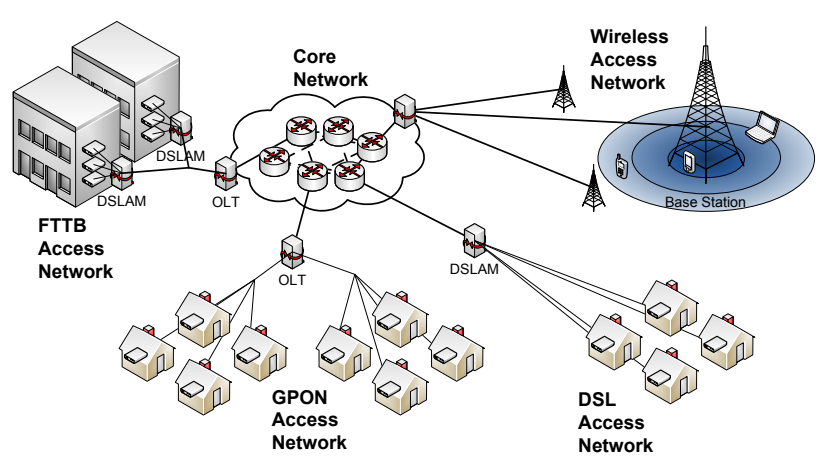

Figure 1. Network Overview

Different research efforts are being taken to analyze the power consumption of telecommunication equipment [3], [4]. Next to the characterization of the power consumption we also give an overview of the optimization strategies. Important work has already been demonstrated in [5] where the authors suggest the introduction of sleep modes and in [6] which suggests component optimization and power management as power saving strategies. However, currently new approaches or variations on the suggested approaches have emerged.

In Section II we introduce the different network architectures and the design parameters that define their power consumption. Based on these parameters the power consumption is then quantified in Section III. In Section IV we elaborate on approaches for the reduction of the power consumption.

\section{Network ARChitectures}

Fig. 1 gives an overview of the different types of network architectures we consider. We make a distinction between fixed line and wireless access networks and core networks.

\section{A. Access Network Architecture}

The purpose of access networks is to provide a connection to the users through which they access the internet. They are usually organized in tree structures. All users are connected to a central office in which the traffic is aggregated and transferred to a core network. This connection is provided through different branches of the tree. Depending on the 
used technologies, the tree has different aggregation levels at intermediary nodes. In the access networks we distinguish between wireless and fixed line access networks (Fig. 1).

In fixed line access networks the user connects through a physical wire. Three main types of technologies are currently used. Firstly there is Digital Subscriber Line (DSL) which uses the twisted pair copper cables from the old telephone lines. Several technology flavours exist, varying in bit rate and maximum range. The ADSL-type (Asymmetric DSL) and VDSL-type (Very high bit rate DSL) flavours are most wellknown.

A second technology is the coax cable technology on which the DOCSIS (Data Over Cable Service Interface Specification) standard is used. These networks are typically built starting from legacy television broadcasting networks.

Currently optical technologies are emerging. These technologies are already used deeper in the network where higher bit rates are required. At present, optical technologies start to appear at the user edge of the network. They are built with either a dedicated connection to the user (point-to-point), an intermediary active splitter (active star) or intermediary passive splitters (passive optical network).

Depending on the offered bit rate and the traffic aggregation the technologies can be used together in an access network. When aggregating the bit rates of VDSL only optical technologies are able to handle the aggregated traffic load. Therefore cable access networks are constructed with an optical backhaul and denoted as Hybrid Fibre Coax (HFC). Also, optical access networks can be terminated with a VDSL node with limited range and are called fibre to the building (FTTB), fibre to the cabinet (FTTC) or, more generally fibre to the $\mathrm{x}$ (FTTx). Note however that FTTx also includes fibre to the home (FTTH), which denotes the full optical access networks.

In this analysis we will focus on the optical and the DSL technologies.

The traffic on the access network is bursty and very variable. The equipment used in the access network on the other hand has a power consumption that is largely constant in time and thus load independent. Therefore, when evaluating power consumption in the access networks, we will consider the power consumption per subscriber as a metric.

ADSL used to be the main access network technology providing downstream speeds from $8 \mathrm{Mbps}$ (ADSL) to $24 \mathrm{Mbps}$ (ADSL2+) and upstream speeds of 1 Mbps. The maximum range is between 1.5 and $5.5 \mathrm{~km}$. The largest range corresponds with the lowest bit rate capacity. This range allows for large numbers of users to be aggregated in the first node of the access network. Because of this large user aggregation, the power consumption per subscriber of the devices in the backhaul of the access network will be negligible.

VDSL uses an extended frequency spectrum compared to ADSL resulting in higher bit rates but lower ranges. Therefore the first aggregation will be closer to the user. This also means that a larger backhaul network will be required and the power consumption of that backhaul network will be more significant.

Optical fibre technologies allow for both higher bit rates and ranges. The bit rate can go up to $10 \mathrm{Gbps}$ for a single optical fibre with a maximum range between 10 and $20 \mathrm{~km}$. Currently, these bit rates are too high for a single subscriber. Therefore, point-to-point connections are mainly used in the backhaul network to aggregate large amounts of traffic. In active star and passive optical network (PON) architectures the bit rate capacity is distributed over large numbers of users. For PON networks split ratios of $32($ range $=20 \mathrm{~km})$ and 64 (range $=10 \mathrm{~km}$ ) are common. The most frequently used standard is GPON (Gigabit PON). Current implementations don't provide large numbers of fibre connections on the optical line terminal (OLT). Usually, between 4 and 72 fibres can be connected.

In wireless access networks the user connection is provided through a wireless link. The user's devices use radio signals to connect to a base station, which is then further connected to the central office through a backhaul network. Different technologies are available varying in transmission power, transmission frequency, modulation scheme and multiplexing technique and thus providing different access bit rates to the users.

Three main emerging wireless technologies are mobile WiMAX (Worldwide Interoperability for Microwave Access), HSPA (High Speed Packet Access), and LTE (Long Term Evolution). Mobile WiMAX is based on the IEEE 802.16 standard. It operates in the $2-6 \mathrm{GHz}$ band and is developed for mobile wireless applications and allows people to communicate while they are moving. The highest supported bit rate is approximately $70 \mathrm{Mbps}$.

HSPA is the successor of the widely deployed UMTS (Universal Mobile Telecommunications System). HSPA provides increased performance by using improved modulation schemes and by refining the protocols by which handsets and base stations communicate. The end-user experience is further improved by increasing the peak data rates up to $14 \mathrm{Mbps}$ in the downlink. HSPA uses the $2.1 \mathrm{GHz}$ band.

LTE is the newest wireless broadband technology. LTE is marketed as the fourth generation $(4 \mathrm{G})$ of radio technologies. Targets for the bit rate are to have peak data rates from $10 \mathrm{Mbps}$ up to $300 \mathrm{Mbps}$ in the downlink. However, in practical implementatios $300 \mathrm{Mbps}$ rates have not yet been achieved. LTE uses the $2.6 \mathrm{GHz}$ band. However, in the future LTE may be using the $700 \mathrm{MHz}$ band.

Contrary to the fixed line access networks, the determining factor in wireless access network design is the area covered by the base stations. The covered area is related to the input power of the base station antenna and the bit rate [3]. The input power determines the reach of the signal and thus the area covered by a base station. Current technologies allow for different modulation schemes being used, determined by the signal to noise ratio. Therefore, higher bit rates will be achieved at smaller ranges from the base station.

At the customer premise the access networks connect to customer premise equipment. For fixed line networks this is usually a home gateway which then further connects to other equipment such as notebook or a settop-box. For wireless access networks this equipment is more diverse. It can be a mobile phone, a wireless network interface card in a computer 


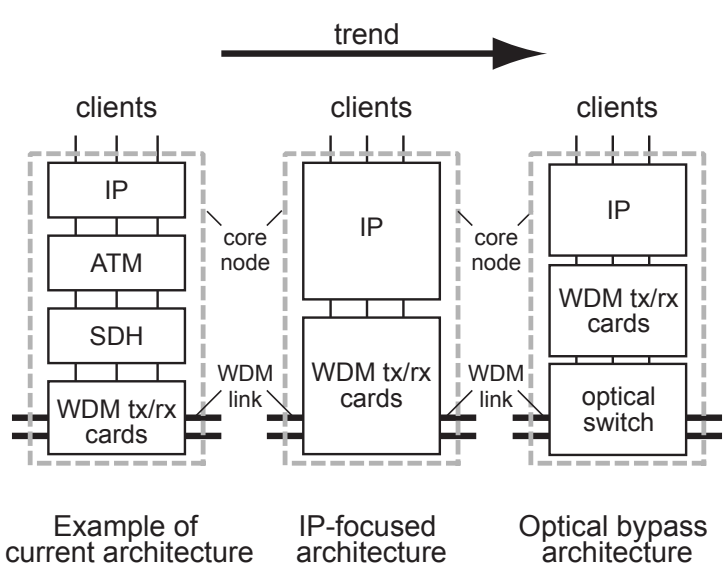

(a) (c)

Figure 2. Core Network Node Architecture

or also a home gateway. For wireless technologies we therefore use the more generic term 'mobile station'.

\section{B. Core Network Architecture}

Access networks aggregate the users in a certain area. In order to interconnect these areas core networks are used. A core network consists of a number of core nodes that are interconnected through wavelength-division multiplexed (WDM) optical fibre links, usually in a mesh or ring topology.

Current core networks are typically a mix of several layers of technologies on top of each other, such as IP-over-ATMover-SDH, as illustrated in Fig. 2(a). However, there is a trend to move to more homogenous architectures where IP is routed directly over WDM links (Fig.2(b)). Given this trend, we will focus on the latter architecture only.

From a high-level view, core nodes are optical-electricaloptical based. This means that all optical traffic is converted to the electronic domain and processed by the node, whether the traffic is terminated at this node or not. In general, a node consists of a number of WDM transmit and receive cards, also referred to as transponders or transceivers, which are connected to an IP router. The IP router in turn can be connected to a number of access routers.

WDM fibre links carry a number of wavelengths, with each wavelength typically having a capacity of $10 \mathrm{Gbps}$ or $40 \mathrm{Gbps}$. 40 to 80 wavelengths per fibre are common. Optical amplifiers are required at intervals of about $80 \mathrm{~km}$ to make up for signal attenuation. With the finite range of light paths (depending on the line rate and technology employed, this is in the order of 1000 to $4000 \mathrm{~km}$ ), long links require regeneration of the optical signal.

\section{Quantification Of Power Consumption}

\section{A. Fixed Line Access Networks}

In fixed line access networks each subscriber (Subs) has a dedicated connection. Thus, the power per subscriber will be a stable metric. In DSL technologies the last node before the subscriber is the DSLAM (DSL Access Multiplexer). ADSL

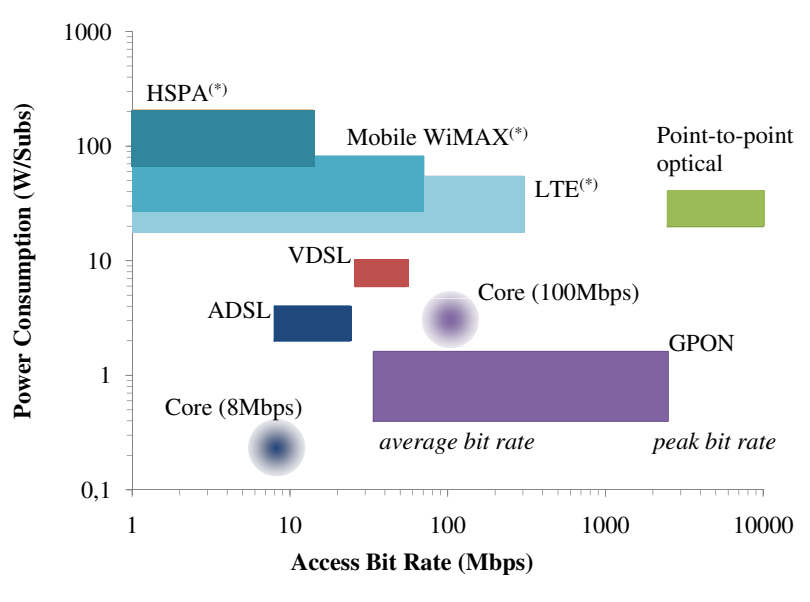

(*) Based on 100 - 300 subscribers / km²

Figure 3. Power Consumption per Subscriber of different Network Technologies

equipment consumes $1-2 \mathrm{~W} / \mathrm{Subs}$, VDSL equipment roughly consumes $3-5 \mathrm{~W} / \mathrm{Subs}$. VDSL equipment power consumption is slightly higher although trends indicate this technology is being optimized.

Optical network equipment currently consumes $10-20 \mathrm{~W}$ per connection. However, using GPON technology this can be further distributed. The OLT consumes $0.2-0.8 \mathrm{~W} / \mathrm{Subs}$. Due to the small range of VDSL technology, it is possible that VDSL equipment with a small number of connections are used. For example, for a VDSL DSLAM with 16 connections the power consumption of the optical backhaul will be $0.01-$ $0.05 \mathrm{~W} /$ Subs.

When evaluating the power consumption of these devices, one needs to consider that the premises in where they are located often needs to be cooled. Also measures are taken in order to prevent power failure. This overhead is expressed in Power Usage Effectiveness (PUE) that denotes the factor by which the equipment is to be multiplied in order to know the total power consumption (i.e. equipment + overhead). The PUE is typically a factor two. This means in reality the above mentioned numbers need to be doubled to estimate the full power consumption.

The power consumptions, including the PUE and the delivered bit rates, are summarized for the different technologies in Table I and Fig. 3.

\section{B. Wireless Access Networks}

In wireless access networks, the highest power consumer is the base station. A base station is here defined as the equipment needed to communicate with the mobile stations and the backhaul network. For the base stations we assume outdoor placement in a suburban environment at a height of $30 \mathrm{~m}$, covering three sectors and a mobile station at a height of $1.5 \mathrm{~m}$.

In order to make a fair comparison between the considered technologies, we define a bit rate per active user of approximately 3 Mbps. We consider the power consumption of the complete base station, which also includes the PUE overhead. 
In wireless access networks, the power per subscriber will be largely dependent on the subscriber density in the covered area. Therefore we first evaluate the power consumed per base station and then translate that to power consumption per user.

Mobile WiMAX has a power consumption of approximately $2.9 \mathrm{~kW}$ per base station, and a range of $340 \mathrm{~m}$. LTE has the highest power consumption, $3.7 \mathrm{~kW}$ per base station, and the largest range, of approximately $470 \mathrm{~m}$. HSPA has the lowest range, $240 \mathrm{~m}$, of all the considered technologies and a power consumption of $3.7 \mathrm{~kW}$ per base station, which is comparable to the power consumption of LTE.

Considering a subscriber density of 300 users per $\mathrm{km}^{2}$ and comparing the power consumption per user, we see that mobile LTE performs the best with a power consumption of $18 \mathrm{~W} / \mathrm{Subs}$, followed by Mobile WiMAX with a power consumption of $27 \mathrm{~W} / \mathrm{Subs}$. The power consumption is lower for LTE because of its large range. HSPA has the highest power consumption per user, $68 \mathrm{~W} / \mathrm{Subs}$, caused by its lower range. Note that these numbers are related to the considered subscriber density. When the subscriber density is halved, the power per subscriber doubles.

Also for wireless access networks the parameters are summarized in Table I and Fig. 3.

\section{Customer Premise Equipment}

Next to the power consumption of the access network, the power consumption of the customer premise equipment is important as well.

Currently, for fixed line technologies, the home gateway (such as a DSL modem) consumes $5-10 \mathrm{~W}$, which is higher than the power consumption in the access network. Home gateways for optical networks also tend to have higher energy consumption than their DSL counterparts. This is a problem since it can annihilate the potential power reduction benefit of adopting GPON technology. In wireless networks the power consumption of the mobile stations is much lower since these are designed for mobile applications, which require low power consumption for long autonomy times.

\section{Core Networks}

As shown in Fig. 4 the major share, about 90\%, of the core network power consumption is concentrated in the nodes. The WDM links, by way of the optical amplifiers, make up only around $10 \%$ or less of the power consumption.

We used datasheets of Juniper T-series core routers to determine the power consumption distribution among the components. The line cards that provide input/output interfaces and that apply the packet forwarding logic are the major power consumers. The line cards' layer 1 and 2 media interface provides framing, line speed signalling and physical connection to a specific network media type such as ATM, SONET/SDH or Ethernet. The purpose of core networks is to transfer traffic streams between different sites. Therefore, energy consumption in the core network is typically expressed in Watts per transferred bit. Fig. 5 plots the maximum power consumption of a number of Juniper routers against their aggregated capacity. As can be seen, high-end routers are

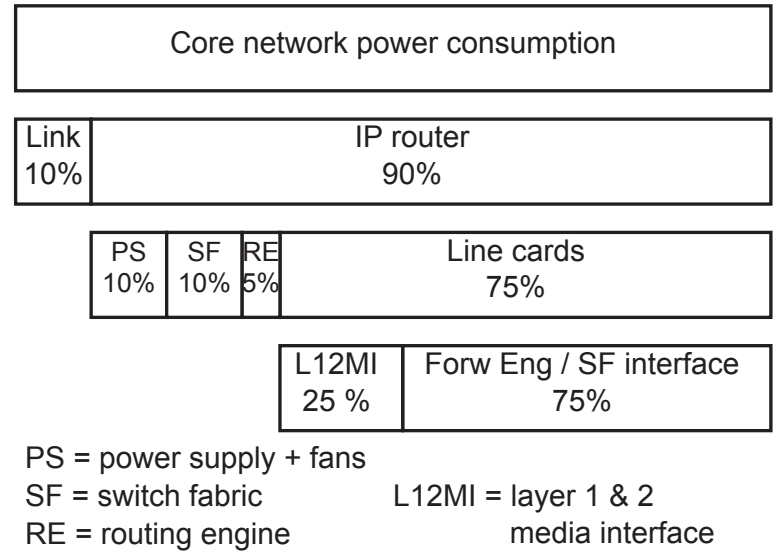

Figure 4. Generalized core network power consumption distribution

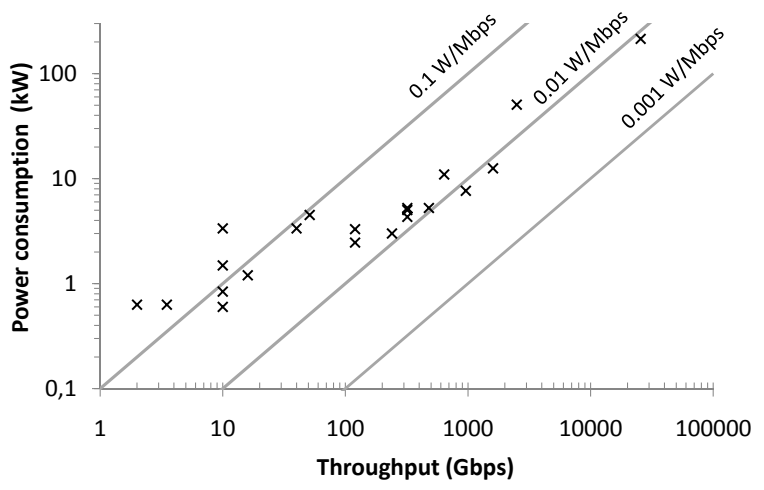

Figure 5. Power consumption of a set of mid-tier and high-end routers

more energy-efficient than low-end routers: while consuming more in absolute values, the power required to transfer a bit decreases with increasing router capacity. Current routers consume between $0.1 \mathrm{~W} / \mathrm{Mbps}$ and $0.01 \mathrm{~W} / \mathrm{Mbps}$. On average, taking into account that near the edge of the core are a higher number of low-end routers, core routers consume about $0.05 \mathrm{~W} / \mathrm{Mbps}$. These values already include a correction for PUE.

In [4] a calculation method is suggested to estimate the power consumption per subsriber of the Internet. Based on this method, we estimate that at ADSL access bit rates ( $8 \mathrm{Mbps})$ the core consumes about $0.24 \mathrm{~W} / \mathrm{subs}$. When accounting for an increase in access bit rate to $100 \mathrm{Mbps}$ this will increase to approximately $3 \mathrm{~W} / \mathrm{subs}$. We have indicated these values in Fig. 3. Currently, the power consumption in core networks is significantly lower than the access network. With increasing bit rates and increasing energy efficiency due to the adoptation of GPON networks the power consumption of core networks will increase.

\section{Power Consumption Optimization}

\section{A. Switching Off Components}

Currently, telecommunications networks are designed to handle the peak loads and little consideration is given to 


\begin{tabular}{|c|c|c|c|c|c|}
\hline Technology & $\begin{array}{l}\text { Range } \\
(\mathrm{km})\end{array}$ & $\begin{array}{l}\text { Bit Rate } \\
\text { (Mbps) }\end{array}$ & Users/Node & $\begin{array}{l}\text { Minimal User Density } \\
\left(\mathrm{subs} / \mathrm{km}^{2}\right)\end{array}$ & $\begin{array}{l}\text { Power/subs (with PUE) } \\
\text { (W/subs) }\end{array}$ \\
\hline$\overline{A D S L}$ & 5.5 & $8(*)$ & \multirow[b]{2}{*}{$384-768$} & $44-8$ & \multirow[b]{2}{*}{$2-4$} \\
\hline ADSL2+ & 1.5 & $24(*)$ & & $50-100$ & \\
\hline \multirow{2}{*}{ VDSL } & 1.0 & $26(*)$ & & $5-60$ & \multirow{3}{*}{$6-10$} \\
\hline & 0.3 & $55(*)$ & $16-192$ & $50-700$ & \\
\hline VDSL2+ & 0.3 & 100 & & $50-700$ & \\
\hline GPON (32) & 20 & $2488 / 32$ & $(4-72) * 32$ & $0.1-2$ & \multirow{2}{*}{$0.4-1.6$} \\
\hline GPON (64) & 10 & $2488 / 64$ & $(4-72) * 64$ & $0.8-14$ & \\
\hline Mobile WiMAX & 0.340 (3 Mbps) & $1-70$ & $272(* *)$ & N/A & $27(* * *)$ \\
\hline HSPA & 0.240 (3 Mbps) & $1-14$ & $225(* *)$ & N/A & $68(* * *)$ \\
\hline LTE & 0.470 (3 Mbps) & $1-300$ & $180(* *)$ & N/A & $18(* * *)$ \\
\hline
\end{tabular}

Table I

PROPERTIES OF DIFFERENT ACCESS NETWORK TECHNOLOGIES

medium and low load situations. Designing adaptable networks, which are able to switch off elements when the demand is lower, will lead to lower power consuming networks.

In core networks this can be achieved with dynamic topology optimization. This means that from the multiple possible topologies that satisfy the required traffic demands, the topologies with lower overall power consumption should be preferred. Dynamic optimization typically exploits the daily or weekly alternations in traffic load, where off-peak volumes are potentially lower than $50 \%$ of peak volumes. When employing multilayer traffic engineering (MLTE) and changing the MLTE strategy to optimize towards power consumption, reductions of more than 50\% during off-peak hours can be achieved [7]. The MLTE strategy adapts the topology to optimize for power consumption, thereby increasing the number of inactive line cards, which can subsequently be switched off and thus save power.

Since access networks are structured in a tree, dynamic topology optimization will not be applicable. In wireless access networks however, optimization can be achieved by utilizing hybrid hierarchical base station deployment. When using base stations with differentiated cell sizes and wireless network technologies, a basic access network can be created providing a low bit rate but high coverage to the users. In the hierarchical layers above, base stations with smaller cell sizes but higher bit rates can be utilized to provide the high bandwidth connections when these are required. The advantage is that the higher layers can be put to sleep and only need to be activated with high traffic demand.

Finally, it is important to optimize the power consumption of the home gateway. These are individual devices that only need to be active during periods where the user is active. At other times, it can in principle be switched off although in reality this rarely happens. In legislation concerning standby power consumption standards of 0.5 Watts are emerging. Implementing this on home gateways will already lead to large optimizations.

\section{B. Reducing Load}

With the idle components in the telecommunication networks switched off, the next step is to reduce the load on the remaining components. This strategy will be especially important in access networks since we already pointed out it is difficult to switch off elements.

Adaptive link rate is a strategy in which different line rates are supported on a link. The lower line rates are assumed to consume less power and thus power can be saved. At the customer premise this can be used to reduce the power consumption of the home gateway. Also in different access network technologies this strategy is showing potential. However, mainly the higher link rates in the order of 1 - 10 Gbps have significantly higher power consumption compared to lower link rates. Secondly, the algorithms for adaptive link rate use larger packet buffers. These larger buffers also require hardware that needs to be powered. In core networks it makes less sense to use adaptive link rates since the traffic shows less variation.

In core networks, a promising technique to reduce power consumption is optical bypass, which is already in use for cost reduction and router capacity offloading (Fig. 2(c)). Traffic not intended for the intermediate node remains in the optical domain and is not processed by the core router. The light path is switched, using optical add/drop multiplexers (OADMs) or optical cross connects (OXCs), from an incoming fibre link This allows to reduce the capacity of the router and the corresponding power consumption. Optical bypass is possible on single-wavelength granularity or on waveband granularity (requiring less ports in the OXC or OADM since multiple wavelengths are switched at the same time). Wavelength conversion can be employed to optimize fibre utilization and resolve contention. Depending on the light path utilization and network size, the power saving potential of optical bypass is up to $45 \%$ [8].

\section{Optimizing Power Consumption of the Remaining Compo- nents}

When the networks are optimized and the load is minimized, the power consumption of the elements should be reduced.

The energy efficiency of wireless access networks can be improved by increasing the ranges of the base stations. Thus, larger areas can be covered by a single base station and less base stations are required. This can be done by using 
multiple transmitting and receiving antennas. This technique is known as MIMO (Multiple Input Multiple Output). When using for example 2 transmitting and 2 receiving antennas, i.e. $2 \times 2$ MIMO, the range increases with $66 \%$, while the power consumption increases only with 2 to $4 \%$ resulting in a higher energy efficiency.

The technique of optical bypass illustrated an evolution from point-to-point WDM networks to more optical circuit-switched networks. Optical burst switching and optical packet switching take this technique a step further and are supposed to provide an even finer switching granularity. In optical packet switching, individual packets are switched optically on the correct outgoing fibre. Because optical buffers with an appropriate size are currently infeasible, optical burst switching is proposed as an intermediate technology. A control signal is send in advance of the packets, and allows the burst-switched router to set up a light path for the soon-to-arrive data, eliminating the need for buffering.

While optical packet switching is thought to provide a lower bound to network power consumption, since it eliminates power-hungry optical-electrical-optical conversions, it is not yet technically feasible [9]. On the other hand, it is argued that with the line card buffers and switch fabric - the two main candidates for optical implementation - consuming only about $15 \%$ of the total power consumption of an electronic router, potential energy savings are not as high as commonly expected [10]. A hybrid approach in which optical switches still use electronic buffering seems a more feasible low-power approach for the next decade.

It is not yet clear if the technique of optical burst switching is a viable alternative, the main issue being the relatively low throughput requiring an overbuild.

For continent-sized core networks, increasing the maximum optical path length (i.e., not requiring regeneration of the optical signal) can reduce power consumption. For a panEuropean network, savings can be up to $10 \%$.

\section{CONCLUSiON}

The number of internet users is fastly increasing and these users demand increasing bit rates. At the same time the carbon footprint of ICT has to be reduced. Saving power in telecommunication networks is becoming an important challenge. Emerging technologies can lead to reduced power consumptions but the design of these technologies needs to be applied with a low power consumption in mind. This means switching off components where possible, reducing the loads on the networks, and optimizing the power consumption of the network elements.

Currently, the main share of that power consumption lies near the customer. In fixed line access techynologies the largest power consumper is the home gateway which can easily be optimized with relatively easy approaches. For wireless access technologies the largest power consumer is the base station.

In fixed line access networks power consumption optimization is not straightforward due to the tree topology of such networks. However, the technology shift towards full optical networks can lead to considerable reductions. In wireless access networks many optimizations can still be implemented. In core networks the power consumption is currently relatively low. Nonetheless, due to the expected increase in traffic volumes, power optimizations are welcomed here as well.

\section{REFERENCES}

[1] M. Pickavet, W. Vereecken, S. Demeyer, P. Audenaert, B. Vermeulen, C. Develder, D. Colle, B. Dhoedt, and P. Demeester, "Worldwide energy needs for ICT: The rise of power-aware networking," in Advanced Networks and Telecommunication Systems, 2008. ANTS '08. 2nd International Symposium on, Dec. 2008, pp. 1-3.

[2] M. Webb et al., "Smart 2020: Enabling the low carbon economy in the information age," The Climate Group, Tech. Rep., 2008.

[3] M. Deruyck, W. Vereecken, E. Tanghe, W. Joseph, M. Pickavet, L. Martens, and P. Demeester, "Power consumption in wireless access networks," in European Wireless, 2010.

[4] J. Baliga, R. Ayre, K. Hinton, W. Sorin, and R. Tucker, "Energy consumption in optical ip networks," Lightwave Technology, Journal of, vol. 27, no. 13, pp. 2391-2403, July 2009.

[5] M. Gupta and S. Singh, "Greening of the internet," in SIGCOMM '03: Proceedings of the 2003 conference on Applications, technologies, architectures, and protocols for computer communications. New York, NY, USA: ACM, 2003, pp. 19-26.

[6] K. Christensen and B. Nordman, "Reducing the energy consumption of networked devices," in IEEE 802.3 tutorial, 2005.

[7] B. Puype, W. Vereecken, D. Colle, M. Pickavet, and P. Demeester, "Power reduction techniques in multilayer traffic engineering," in Transparent Optical Networks, 2009. ICTON '09. 11th International Conference on, July 2009, pp. 1-4.

[8] W. Van Heddeghem, M. De Groote, W. Vereecken, D. Colle, M. Pickavet, and P. Demeester, "Energy-efficiency in telecommunications networks: Link-by-link versus end-to-end grooming," in Optical Network Design and Modeling, 2010. ONDM '10. 14th Conference on, Kyoto, Japan, February 2010.

[9] S. Aleksic, "Analysis of power consumption in future high-capacity network nodes," Optical Communications and Networking, IEEE/OSA Journal of, vol. 1, no. 3, pp. 245-258, August 2009.

[10] R. Tucker, R. Parthiban, J. Baliga, K. Hinton, R. Ayre, and W. Sorin, "Evolution of wdm optical ip networks: A cost and energy perspective," Lightwave Technology, Journal of, vol. 27, no. 3, pp. 243 -252, Feb. 2009. 